Proc. XII Int. School on Theoretical Physics - Symmetry and Structural Properties of Condensed Matter

\title{
The Influence of Potential Energy Shape on the Total Electrical Efficiency of the Energy Harvester
}

\author{
K. KuCAB* AND G. Górski
}

Faculty of Mathematics and Natural Sciences, University of Rzeszów, S. Pigonia 1, 35-310 Rzeszów, Poland

\begin{abstract}
In this theoretical work we analyze the total effective electric power versus base acceleration amplitude generated by the energy harvesting system with an electromagnetic transducer. We compare the results for both linear and nonlinear case. The transition from linear to nonlinear behavior of the system can be achieved by the change of device geometry. To improve the power efficiency of our device we also examine the dependence of crossover point of acceleration amplitudes where generated power in the nonlinear system starts to exceed the generated power in the linear regime. We have found that the crossover point can be moved towards relatively small base acceleration values by appropriate selection of system nonlinearity "strength".
\end{abstract}

DOI: 10.12693/APhysPolA.132.118

PACS/topics: 05.10.-a, 88.05.-b, 41.20.Gz

\section{Introduction}

The problem of acquiring an energy from the environment is actually in a great interest. The modern electronic devices, e.g. wireless sensors used for example for structural health monitoring applications, are widely used. These devices are characterized by very small power consumption. Due to this fact the problem of their power supply can be reduced to the "self-powering" problem. This is because the maintenance cost of replacing batteries in this type of sensors can exceed the sensors' cost. The energy harvesting devices can also play a crucial role especially in hard to reach environments [1-4]. As we know, the linear systems subjected to the harmonic excitations give the high energies only at one frequency, called the resonance frequency. This fact restricts the use of such systems in a real life, where the broad range of base acceleration frequency spectrum is observed. Constructing the efficient energy harvesting device one should take into account that the energy harvesters should have a broad band of frequencies for which the power generated is relatively large [5]. As many authors show, to extend the frequency range where the effective power is relatively large one can use the nonlinear systems (see e.g. [6-10]). In many papers authors consider the energy harvesting devices based on electromotive force phenomena [11-13]. The voltage generated in these systems is produced by the electromagnetic transducer. One can use the coil inside which the small magnet vibrates. The quasi-periodic movement of this magnet takes place in the magnetic field generated by fixed magnets or additionally in the presence of the springs attached to the moving magnet and the static ones. The presence of magnetic field generated by the static magnets causes that the system behaves in a nonlinear way.

*corresponding author; e-mail: kkucab@ur.edu.pl
Lee et al. [14] examined (theoretically and experimentally) the electromagnetic system similar to our device. In the nonlinear case they used the three-magnet device, where the center magnet levitated between fixed ones. The authors also used in the model approximate expression (fifth order fit) for the dependence of magnetic force versus displacement between magnets, while in our work we used the integral relations. The authors analyzed the frequency response of the linear and nonlinear system. They have shown that the nonlinear harvester has significantly higher bandwidth compared to the linear one. Mann and Sims [15] also investigated the harvester that uses magnetic levitation. This model was analysed for harmonic base excitations. The authors state that "engaging the nonlinear response of the system can result in relatively large oscillations over a wider range of frequencies".

In this paper we use the energy harvesting device with electromagnetic transducer. We compare the effective output power generated by this device in two regimes: linear and nonlinear. In the linear domain we will use the Hooke law only. In the nonlinear regime we will use the influence of both the magnetic field and Hooke potential of the springs.

\section{The model}

The scheme of considered device is presented in Fig. 1. The more detailed description of similar system geometry is presented in our previous work [16].

The device can be attached to the vibrating base. The base vibrations are denoted by $z(t)$. The vibrations are transferred by two springs to the center magnet which vibrates inside the coil connected to the electric load $R_{L}$. The role of the springs is twofold. First, they limit the movement of moving magnet which prevents the magnets to contact each other. Secondly, the compressed spring acts on the moving magnet as a stopper. The effects of friction are neglected for simplicity. The dimensions of the device are as follows: static magnet height $-5 \mathrm{~mm}$, moving magnet height $-2.5 \mathrm{~mm}$, diameter of magnets $-30 \mathrm{~mm}$, the device height $-50 \mathrm{~mm}$. In 


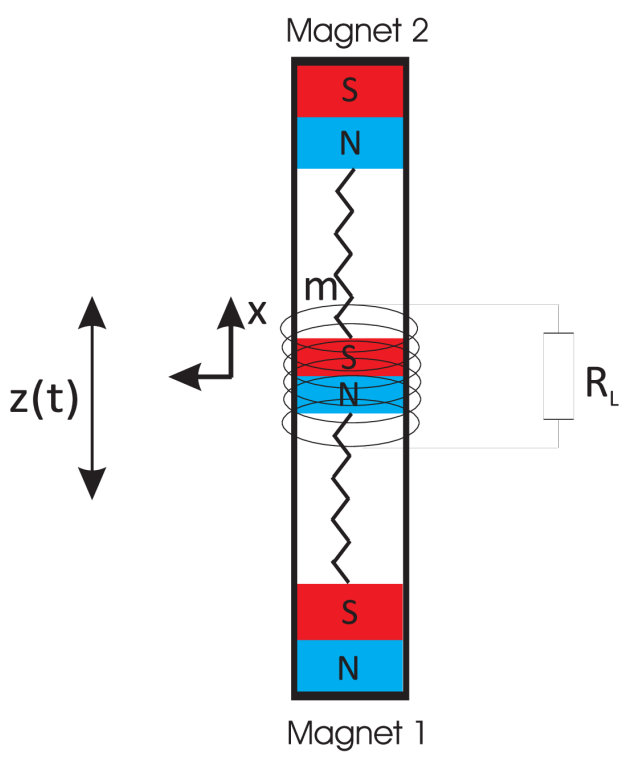

Fig. 1. The energy harvesting device considered by us in the nonlinear configuration. In the linear case the magnets Magnet 1 and Magnet 2 are replaced by the non-magnetic material.

our computations we used the properties of neodymium iron boron $36 / 26$ permanent magnet with magnetization $M=925550 \mathrm{~A} / \mathrm{m}$ (at the temperature $T=20^{\circ} \mathrm{C}$ ). We use the standard Newton and Kirchhoff law in the form that couples the relative displacement of the moving magnet $y(t)=x(t)-z(t)$ with the current $I(t)$ flowing through the electric load

$$
\begin{aligned}
& -\ddot{z}(t)=\ddot{y}(t) \\
& +\frac{1}{m}\left(c \dot{y}(t)-k y(t)-\chi I(t)+F_{\operatorname{magn}}(y)\right),
\end{aligned}
$$

and

$$
L \dot{I}(t)+R I(t)-\chi \dot{y}(t)=0,
$$

where $x(t)$ is the displacement of the moving magnet with respect to its equilibrium position. The resistance $R$ is the sum of internal and external resistive load part, $R=R_{L}+R_{\text {int }}, k=2 k_{0}$, where $k_{0}$ is the stiffness, $\kappa$ is the coefficient related to the mechanical energy dissipation, $\chi$ is related to the transducer properties, and $L$ is the inductance of the coil. The coil dimensions are: height $-10 \mathrm{~mm}$, number of turns -100 , inductance $-1 \mathrm{mH}$, resistance $-22 \Omega$. We use the relation describing the interaction between magnets in the form

$$
F_{\text {magn }}(y)=I_{1}(y)-I_{2}(y) \text {, }
$$

where $I_{1}$ and $I_{2}$ are the integrals related to the device geometry (see e.g. [16, 17]). We also assume that the base acceleration has the simple, sinusoidal form

$$
\ddot{z}(t)=A_{0} \sin \omega t,
$$

where $A_{0}$ is its amplitude (expressed in $g$ ).

\section{Results}

We perform the computations using the parameters presented in Table I.

\section{TABLE I}

The values of parameters used in our model.

\begin{tabular}{c|c|c}
\hline$R_{L}=2000 \Omega$ & $R_{\text {int }}=787 \Omega$ & $L=12.5 \mathrm{mH}$ \\
$k_{0}=380-550 \mathrm{~N} / \mathrm{m}$ & $c=5.4 \mathrm{~g} / \mathrm{s}$ & $\chi=5 \mathrm{~N} / \mathrm{A}$
\end{tabular}

To examine the influence of potential energy shape on the energy spectrum and total electrical efficiency of the energy harvester we will change the value of stiffness.

In Fig. 2 we present the potential energy vs. moving magnet distance from its equilibrium position for different values of stiffness.

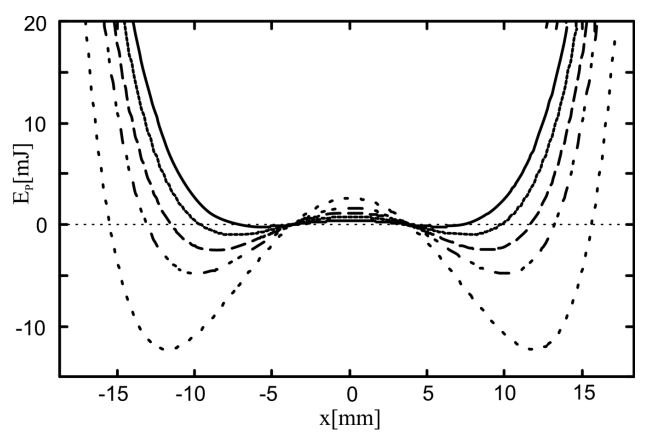

Fig. 2. The potential energy $E_{p}$ versus moving magnet distance $x$ from its equilibrium position for different values of $k_{0}$. The values of $k_{0}$ are: 387 (solid line), 413, 442,473 , and $544 \mathrm{~N} / \mathrm{m}$ (dotted line).

As one can see in Fig. 2, we can affect the "strength" of system nonlinearity by changing the stiffness. To examine the influence of potential shape on generated electric power in the whole range of excitations considered by us, we introduce the effective output power $\langle\bar{P}\rangle$. To obtain $\langle\bar{P}\rangle$ we average the mean output power $\bar{P}=\left\langle I^{2}\right\rangle R_{L}$ over the frequency of excitations, $\omega$, varying from 0 to $3 \omega_{0}$, where $\omega_{0}$ is the resonance frequency. The results showing the influence of base acceleration amplitude $A_{0}$ on $\langle\bar{P}\rangle$ for different values of stiffness are presented in Fig. 3.

As one can see in Fig. 3, the effective output power grows with increase of the base acceleration. Additionally, for some range of $A_{0}$ values, $\langle\bar{P}\rangle$ in the nonlinear case has higher values compared to the linear regime. It is worth to note in here that one of the main goals in the design process of the energy harvesting device is to obtain its high power efficiency for relatively small vibrations (acceleration amplitudes) because in the real world high values of acceleration are dangerous for humans and devices. The crossover point of acceleration amplitudes where generated power in the nonlinear system starts to exceed the generated power in the linear regime can be moved towards relatively small base acceleration values by appropriate selection of device parameters (the stiffness in our case). The value of crossover point versus stiffness can help us to estimate the strength of vibrations where the harvester works in an efficient way. This dependence is presented in Fig. 4. 

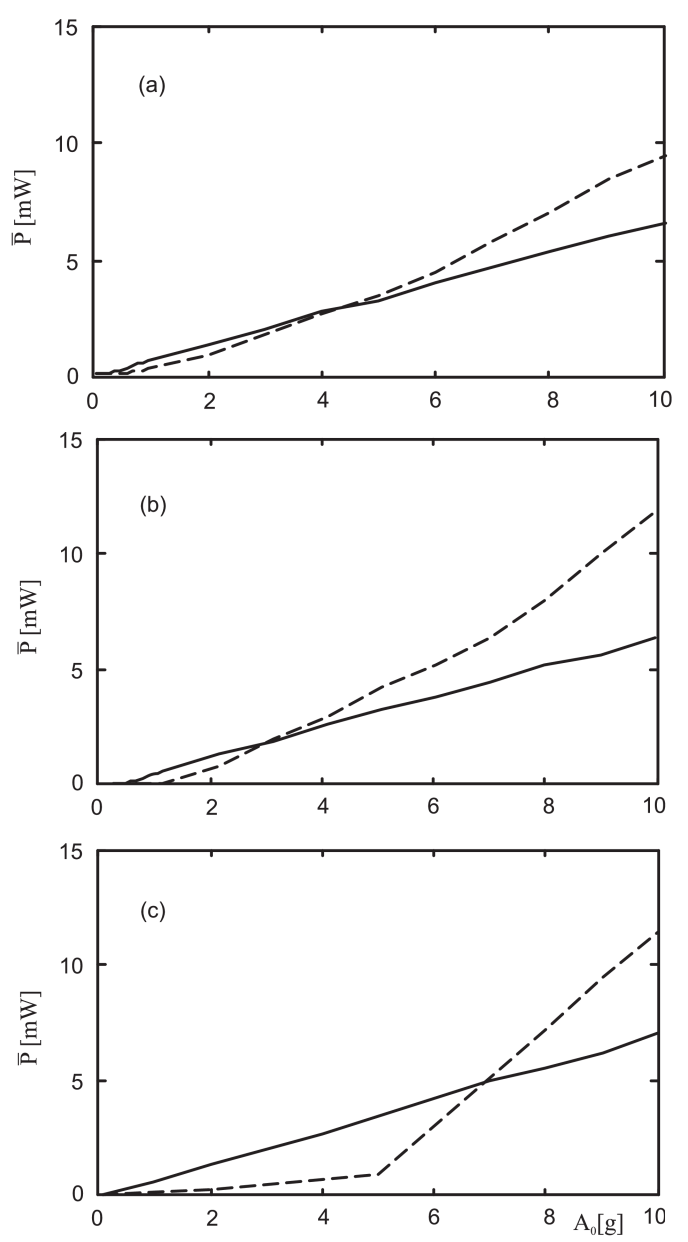

Fig. 3. The effective output power versus base acceleration amplitude for different values of stiffness. (a) $k_{0}=387 \mathrm{~N} / \mathrm{m}$, (b) $k_{0}=413 \mathrm{~N} / \mathrm{m}$, (c) $k_{0}=544 \mathrm{~N} / \mathrm{m}$. Solid line - linear case, dashed line - nonlinear case.

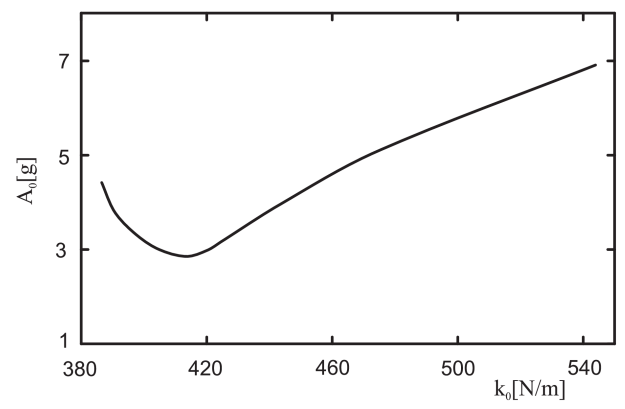

Fig. 4. The acceleration crossover point versus stiffness.

As one can see in Fig. 4 the device works optimally (relatively high output power for small base accelerations) when the stiffness is equal to $k_{0}=413 \mathrm{~N} / \mathrm{m}$.

The computations performed by us were done using Mathematica package. We have used the NDSolve numerical differential equation solver. All the average values were obtained using the Monte Carlo method with number of steps of the order of one hundred million.

\section{Conclusions}

We analyzed the effective output power versus base acceleration amplitude for the energy harvesting device with different degree of nonlinearity. In the nonlinear case, at small acceleration amplitudes, the total harvested power is smaller than the power obtained in the linear case. For some value of acceleration the power in the nonlinear system becomes higher than in the linear case. This crossover point of acceleration has the minimum value for a specific value of stiffness, i.e. for the specific "strength" of system nonlinearity.

\section{Acknowledgments}

This work has received support from Faculty of Mathematics and Natural Sciences, Rzeszów University within the project No. WMP/GD-23/2016, and partial support from Center for Innovation and Transfer of Natural Sciences and Engineering Knowledge at the Rzeszów University.

\section{References}

[1] S.P. Beeby, M.J. Tudor, N.M. White, Measur. Sci. Technol. 17, R175 (2006).

[2] K.V. Selvan, M.S.M. Ali, Renew. Sust. Energ. Rev. 54, 1035 (2016).

[3] H. Sodano, D. Inman, G. Park, The Shock and Vibration Digest 36, 197 (2004).

[4] H. Sodano, D. Inman, G. Park, J. Intell. Mater. Syst. Struct. 16, 67 (2005).

[5] S.C. Stanton, C.C. McGehee, B.P. Mann, Physica D 239, 640 (2010).

[6] R.L. Harne, K.W. Wang, Smart Mater. Struct. 22, 023001 (2013).

[7] W. Martens, U. von Wagner, G. Litak, Eur. Phys. J. Spec. Top. 222, 1665 (2013).

[8] F. Cottone, H. Vocca, L. Gammaitoni, Phys. Rev. Lett. 102, 080601 (2009).

[9] K. Kucab, G. Górski, J. Mizia, Eur. Phys. J. Spec. Top. 222, 1607 (2013).

[10] L. Haitao, Q. Weiyang, L. Chunbo, D. Wangzheng, Z. Zhiyong, Smart Mater. Struct. 25, 015001 (2016).

[11] B.P. Mann, B.A. Owens, J. Sound Vibrat. 329, 1215 (2010).

[12] M. Borowiec, G. Litak, S. Lenci, in: Discontinuity and Complexity in Nonlinear Physical Systems, Eds. J.A. Tenreiro Machado, D. Baleanu, A.C.J. Luo, Springer, New York 2014, p. 315.

[13] S. Kwon, J. Park, K. Law, Smart Mater. Struct. 22 , 055007 (2013).

[14] Ch. Lee, D. Stamp, N.R. Kapania, J.O. MurMiranda, Proc. SPIE 7683, $76830 \mathrm{Y}$ (2010).

[15] B.P. Mann, N.D. Sims, J. Sound Vibrat. 319, 515 (2009).

[16] K. Kucab, G. Górski, J. Mizia, Eur. Phys. J. Spec. Top. 224, 2909 (2015).

[17] D. Vokoun, M. Beleggia, L. Heller, P. Šittner, J. Magn. Magn. Mater. 321, 3758 (2009). 